\title{
Anemia and iron, zinc, copper and magnesium deficiency in Mexican adolescents: National Health and Nutrition Survey 2006
}

\author{
Vanessa De la Cruz-Góngora, MsC,(I) Berenice Gaona, MsC,(I) Salvador Villalpando, MD, PhD,(I)
} Teresa Shamah-Levy, MsC, (I) Ricardo Robledo, PhD. (I)

\author{
De la Cruz-Góngora V, Gaona B, Villalpando S, \\ Shamah-Levy T, Robledo R. \\ Anemia and iron, zinc, copper and magnesium \\ deficiency in Mexican adolescents: \\ National Health and Nutrition Survey 2006. \\ Salud Publica Mex 20 12;54:135-145.
}

\begin{abstract}
Objetive. To describe the frequency of anemia and iron, zinc, copper and magnesium deficiencies among Mexican adolescents in the probabilistic survey ENSANUT 2006. Materials and methods. The sample included 2447 adolescents aged 12 to 19 y. Capillary hemoglobin and venous blood samples were collected to measure the concentrations of ferritin, sTFR, CRP, zinc, iron, copper and magnesium. Logistic regression models were constructed to assess the risk for mineral deficiencies. Results. The overall prevalence of anemia was II 1.8 and $4.6 \%$, body iron deficiency 18.2 and $7.9 \%$ for females and males, respectively. Overall prevalence of tissue iron deficiency was $6.9 \%$, low serum copper were 14.4 and $12.25 \%$; zinc 28.4 and $24.5 \%$, magnesium 40 and $35.3 \%$; for females and males, respectively. Conclusions. There is a high prevalence of mineral deficiency in Mexican adolescents; females were more prone to have more mineral deficiencies. Nutritional interventions are necessaries in order to reduce and control them.
\end{abstract}

Keywords: iron; zinc; copper; magnesium; Mexican adolescents
De la Cruz-Góngora V, Gaona B, Villalpando S,

Shamah-Levy T, Robledo R.

Anemia y deficiencia de hierro,

zinc, cobre y magnesio en adolescentes

mexicanos: resultados de la ENSANUT 2006.

Salud Publica Mex 20 12;54:135-I45.

\section{Resumen}

Objetivo. Describir la prevalencia de anemia y deficiencia de hierro, zinc, cobre y magnesio en adolescentes mexicanos en la encuesta probabilística ENSANUT 2006. Material y métodos. La muestra incluyó 2447 adolescentes de 12 a 19 años de edad. Se tomó hemoglobina capilar y muestras de sangre venosa para medir las concentraciones séricas de ferritina, sTFR, CRP, zinc, hierro, cobre y magnesio. Se construyeron modelos de regresión logística para evaluar el riesgo de deficiencia de minerales. Resultados. La prevalencia de anemia fue de $11.8 \%$ en mujeres y $4.6 \%$ en hombres. Las deficiencias de hierro fueron de 18.2 y $7.9 \%$ La deficiencia tisular de hierro fue $6.9 \%$; la baja concentración de cobre fue de 14.4 y $12.25 \%$ la de zinc de 28.4 y $24.5 \%$, la de magnesio fue 40 y $35.3 \%$ en mujeres y hombres, respectivamente. Conclusiones. Existe una alta prevalencia de deficiencia de minerales en los adolescentes; las mujeres tuvieron mayor riesgo. Son necesarias intervenciones nutricionales para reducir o controlar estas deficiencias.

Palabras clave: hierro; zinc; cobre; magnesio; adolescentes mexicanos

(I) Instituto Nacional de Salud Pública. Cuernavaca, Morelos, México.

Received on: April 18, 2011 • Accepted on: August 24, 201I

Corresponding author: Dr. Salvador Villalpando. Instituto Nacional de Salud Pública.

Av. Universidad 655, Col. Santa María Ahuacatitlán. 62100 Cuernavaca, Morelos, México. E-mail: svillalp@insp.mx 
$\mathrm{T}_{\mathrm{t}}$ he deficiency of micronutrients is a health issue far from being resolved globally. ${ }^{1}$ Some mineral deficiencies as such iron and zinc impact negatively on physical growth, immune response, cognitive and emotional development, among others. ${ }^{2}$

There is limited information on the mineral status of copper and magnesium in adolescents. In adult German population, prevalence of magnesium deficiency reached $14.5 \% .{ }^{3}$ Knovich et al reported an association between serum copper levels and anaemia in American population (NHANES II). ${ }^{4}$ Reports on the prevalence of anemia, iron and zinc deficiency are available for several groups of adolescents, ${ }^{5-7}$ varying from $2 \%$ to $5 \%$ for zinc deficiency in British, $43 \%$ in Ethiopia and over 50\% in Sri Lanka population. Also were found $24 \%$ of iron deficiency in Sri Lanka. The Mexican National Nutrition Survey -1999 (NNS-99), described the prevalences of anemia, iron, zinc and iodine status in women between 12 and 49 years of age. The overall prevalence of anemia was $20.8 \%,{ }^{8}$ iron deficiency was $40 \%$ and zinc deficiency $30 \% .{ }^{9}$ However no distinctions were made for adolescents. No information is available for cooper and magnesium deficiencies in population-based surveys in Mexico. The objective of the present analysis is to describe the frequency and distribution of anemia and iron, zinc, copper and magnesium deficiencies among Mexican adolescents, aiming to identify opportunities for intervention.

\section{Population and methods}

Study population. Information for the present analysis was extracted from the dataset of the Mexican National Health and Nutrition Survey of 2006 (ENSANUT 2006). This is a probabilistic survey, representative at the national, regional, urban and rural levels.

A detailed description of the design and sampling procedures was published elsewhere. ${ }^{10}$ Demographic and socioeconomic information was collected using ad hoc questionnaires. Ethnicity was classified if an indigenous language was spoken. Localities with less than 2500 inhabitants were considered as rurals. A socioeconomic index was constructed based on the household characteristics and family assets by a principal component analysis, the index was divided into tertiles being 1 the lowest. The country was divided in four geographic regions: Northern, Center, Mexico City and Southern. Anthropometric information (weight and height) was collected using validated and standardized methods. ${ }^{11,12}$ Body Mass Index (BMI) was computed based on height and weight. ${ }^{13}$

This sample ( $n=2447)$, was extracted from the general dataset of the ENSANUT 2006 including 12-19 yrs of age with a blood sample available.

\section{Methods for serum mineral concentrations and $\mathrm{C}$ reactive protein}

Venous blood samples were drawn and centrifuged at $268 \mathrm{~g}$, in situ. Serum was separated and stored in coded cryovials, preserved in liquid nitrogen until delivery to a central laboratory in Cuernavaca, Mexico.

The serum concentrations of ferritin, soluble transferrin receptors (sTfR) were measured by immunoassay method using commercial kits (Dade Behring Inc). C reactive protein (CRP) was measured by nephelometry, using ultra sensitive monoclonal antibodies (Behring Nephelometer 100 Analyzer).

Low iron stores (LIS) was defined if serum concentrations of ferritin $<12 \mu \mathrm{g} / \mathrm{L}$; tisssue iron deficiency (TID) was defined if serum concentrations of sTfR were $>6 \mathrm{mg} / \mathrm{L}$.

The total body iron (TBI) $(\mathrm{mg} / \mathrm{kg})$ was calculated based on the concentrations of sTfR and s-ferritin using the Cook's equation. ${ }^{14}$

The serum concentrations of iron, zinc, copper and magnesium were measured by inductively coupled plasma atomic emission spectroscopy using a Varian Vista Pro CCD spectrometer.

Zinc deficiency was defined if $<65 \mu \mathrm{g} / \mathrm{dL} .{ }^{15} \mathrm{Low}$ serum copper (LSC) if $<70 \mu \mathrm{g} / \mathrm{dL}$ for males or $<80 \mu \mathrm{g} /$ $\mathrm{dL}$ for females ${ }^{4}$ and low serum magnesium if $<1.82 \mathrm{mg} /$ $\mathrm{dL}(<0.75 \mathrm{mmol} / \mathrm{L}) .{ }^{16}$

\section{Anemia}

Capillary hemoglobin was measured using a portable photometer. Anemia was defined when hemoglobin concentration $(\mathrm{Hb})$ adjusted by altitude was $<120 \mathrm{~g} / \mathrm{L}$ for females or $<130 \mathrm{~g} / \mathrm{L}$ for males. ${ }^{17}$ Iron deficiency anemia (IDA) was defined if an anormal $\mathrm{Hb}$ value coexisted with at least one of the following abnormalities: s-ferritin, sTfR or serum iron. Others causes of anemia were defined when they did not meet the above criteria.

\section{Dietary intake}

Obtained using food-frequency questionnaire (FFQ) collecting information on the seven days prior to the survey, including 101 food items. The validation of the FFQ was published elsewhere. ${ }^{18}$ The intake of energy and macronutrients was computed; the intake in g/day was transformed into total energy, macro and micronutrients using the food composition tables compiled by Instituto Nacional de Salud Pública, combining the USDA Food Composition Tables, and other tables from Mexico and Latin America. ${ }^{18}$ The adequacy was referred 
to the Dietary Reference Intakes (DRI) of the Institute of Medicine of USA. ${ }^{19}$

\section{Statistical analysis}

The characteristics of the sample are described as frequencies and 95\% confidence intervals, stratified by sex. The distribution of TBI, age and sex interactions were evaluated through multiple linear regression models. Logistic regression models were constructed to test the risks for low tissue iron, low serum zinc, copper and magnesium. Heterogeneity of effects by sex and age were tested adjusted by Socioeconomic Status (SES), BMI, CRP, dwelling, geographical regions, ethnicity and being beneficiary of Oportunidades.

Statistical significance was set at $\alpha=0.05$ and $\alpha=0.1$ for interactions. All analyses were adjusted for the sampling design of the survey, using STATA SE v11.

The protocol was approved by the Research, Ethics, and Biosecurity Comitee of Instituto Nacional de Salud Pública, Mexico. Individual assents and informed consent letters were obtained from the parents of all participants.

\section{Results}

This analysis includes 2447 adolescents. The characteristics of the sample are presented in Table I. Briefly, the mean age was $15.1 \mathrm{y}$, with a proportion male/female $45.9 / 54.1 ; 34.1 \%$ lived in rural areas, $7.1 \%$ were of Indian ethnicity and $35.4 \%$ were overweight/obese (Table I).

\section{Prevalence of anemia}

The overall prevalence of anemia was $8.5 \%$, greater in females $(11.8 \%)$, than in males $(4.6 \%)$.

The geometric mean of s-ferritin was lower in the anemic than in the non anemic subjects $(18.32 \mu \mathrm{g} / \mathrm{L}$ vs $32.14 \mu \mathrm{g} / \mathrm{L}$, respectively, $p<0.001)$. On the contrary, the geometric mean of serum copper in anemic was significantly higher than in non anemic subjects (104.5 $\mu \mathrm{g} / \mathrm{dL}$ vs $97.4 \mu \mathrm{g} / \mathrm{dL}$, respectively, $p=0.018$ ) (data not shown). Iron deficiency was present in $41.6 \%$ of all cases of anemia. IDA was greater in anemic females than in males (43.5 vs $36.1 \%$, respectively).

\section{Low iron stores}

The geometric mean of s-ferritin was greater in males than in females $(39.94 \pm 1.03 \mu \mathrm{g} / \mathrm{L}$ vs $24.73 \pm 1.04 \mu \mathrm{g} / \mathrm{L}$ respectively). The overall prevalence of LIS was higher in females than in males ( $18.2 \%$ vs $7.9 \%$, respectively) (Table II).
In a logistic model, age and gender had a differential effect on LIS $(\mathrm{OR}=1.36, p<0.001)$. The risk increased in females for each year of age $(\mathrm{OR}=1.11, p=0.017)$; in males the risk was lower with increasing age $(\mathrm{OR}=0.82$, $p=0.019$ ). No associations were found with dwelling, geographical distribution, SES or ethnicity (Table IV).

\section{Tissue iron deficiency}

The geometric mean concentration of sTfR was $3.58 \pm 1$ $\mathrm{mg} / \mathrm{L}$ with no significant differences by sex. The overall prevalence of TID was $6.96 \%$ (Table II).

In a logistic model, age and gender had a differential effect on TID $(\mathrm{OR}=2.43, p<0.001)$. Age was a higher risk for females (OR=1.19, $p=0.003)$, but protective in males $(\mathrm{OR}=0.5, p<0.001)$. Higher SES tended to be protective and higher BMI was a risk for TID $(\mathrm{OR}=1.05, p=0.022)$. No significant differences were found by dwelling, ethnicity, geographical regions, or affiliation to Oportunidades (Table IV).

\section{Dietary iron intake}

The overall median daily intake of hem $(0.26 \mathrm{mg} / \mathrm{d})$ and non-hem $(10.88 \mathrm{mg} / \mathrm{d})$ iron were above the recommended intake. The overall median adequacy for iron intake was $157.3 \%$; $181.5 \%$ for males and $136.9 \%$ for females. The adequacy of folate and vitamin B12 intake was $70.2 \%$ and $110 \%$ respectively. The daily intake of enhancers of iron absorption as such as ascorbic acid (vitamin C) had an adequacy of $120 \%$, the intake of inhibitors as such phytic acid was very high (1 $240 \mathrm{mg} / \mathrm{d})$.

\section{Total body iron}

The adjusted mean of the calculated TBI was lower in females $(5.58 \pm 0.15 \mathrm{mg} / \mathrm{kg})$ than in males $(7.07 \pm 0.13 \mathrm{mg} /$ $\mathrm{kg}, p<0.001)$. The distribution of TBI was different by sex (K-Smirnof p-value $<0.001)$. A total exhaustion of TBI $(\mathrm{TBI}=\leq 0)$, was more frequent in females $(9.12 \%)$ than in males $(4.82 \%, p=0.002)$.

The TBI increased with age in males (coefficient= $0.66 \mathrm{mg} / \mathrm{kg} ; p<0.001)$; while it decreased in females (coefficient $=-0.15 \mathrm{mg} / \mathrm{kg}, p=0.023$ ), adjusted by CRP (Figure 1).

\section{Low serum copper}

The mean concentration of serum copper was lower in males $(93.23 \pm 1.01 \mu \mathrm{g} / \mathrm{dL})$ than in females $(102.9 \pm 1.01$ $\mu \mathrm{g} / \mathrm{dL})$.

The overall prevalence of low serum copper was $14.14 \%$; with not significant differences by sex. The 
Table I

DesCRIPTION OF THE SAMPLE OF ADOLESCENTS. ENSANUT 2006*

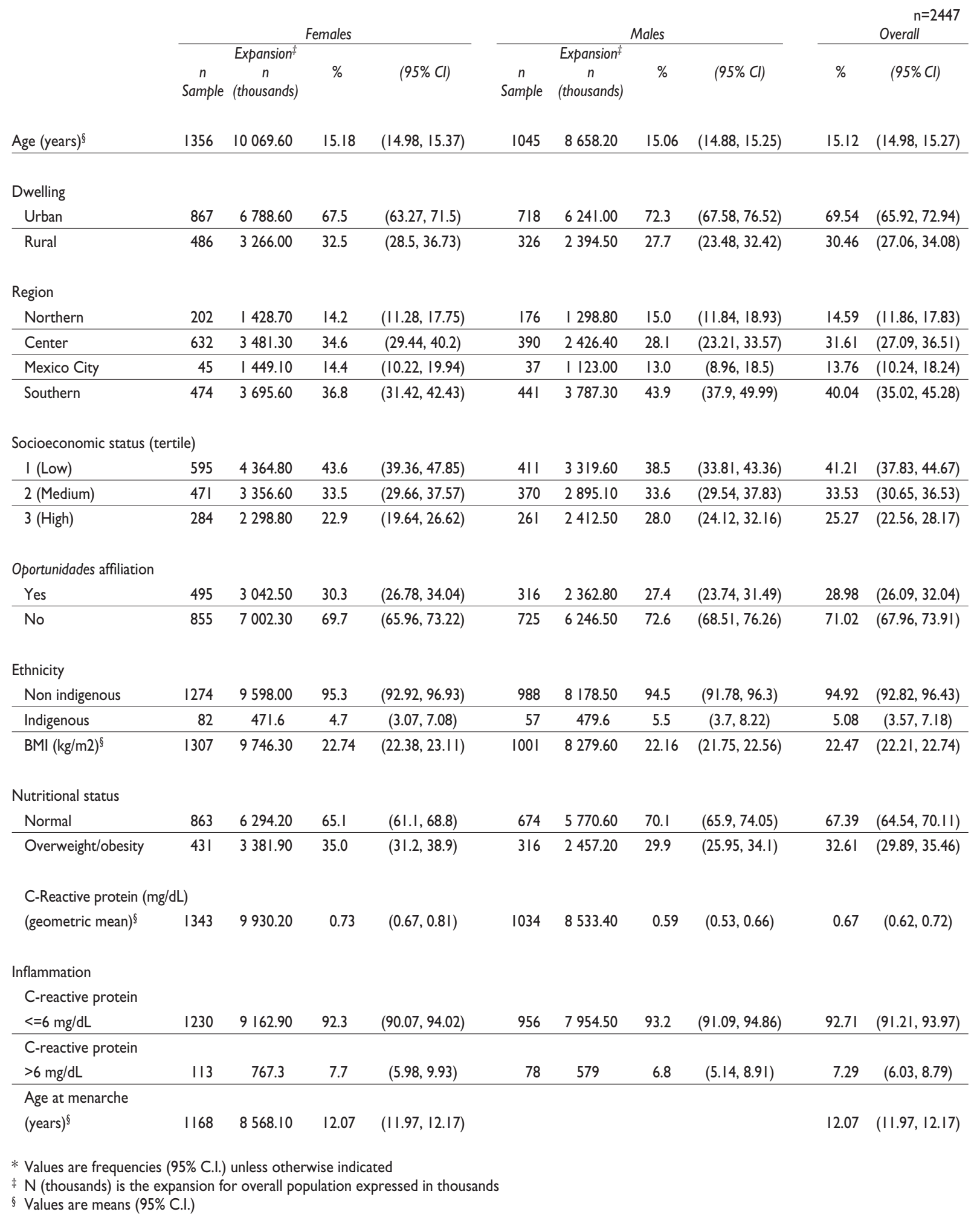


Table II

Prevalence of IRon deficiency measured through ferritin SERUM AND SOLUble tRansferrin ReCePtors CONCENTRATIONS. ENSANUT 2006

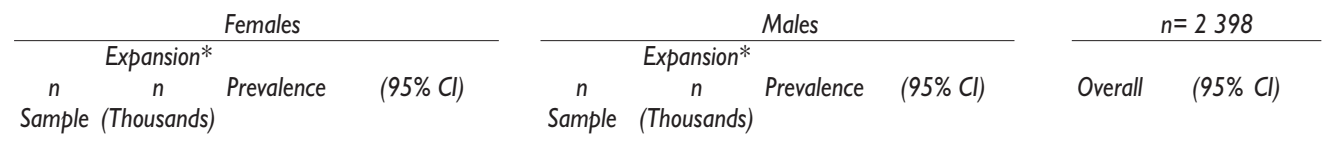

Iron deficiency measured as serum ferritin $<12$ ug/L

\begin{tabular}{|c|c|c|c|c|c|c|c|c|c|c|}
\hline Overall national & 1353 & 9903.8 & 18.24 & $(15.16,21.33)$ & 1045 & 8538.8 & 7.9 & $(5.70,10.07)$ & 13.45 & $(11.52,15.65)$ \\
\hline \multicolumn{11}{|l|}{ Age group } \\
\hline $12-14$ & 619 & 4254.1 & 14.48 & $(10.95,18.01)$ & 503 & 4067.2 & II & $(7.45,14.53)$ & 12.77 & $(10.11,15.43)$ \\
\hline $15-19$ & 734 & 5649.7 & 21.07 & $(\mathrm{I} 6.34,25.8 \mathrm{I})$ & 542 & $447 I .6$ & 5.07 & $(2.56,7.57)$ & 14.01 & $(10.88,17.12)$ \\
\hline \multicolumn{11}{|l|}{ Dwelling } \\
\hline Rural & 479 & 3200.3 & $|5.5|$ & (II.18, 19.8) & 324 & 2359.0 & 9.31 & $(5.4,13.22)$ & 12.88 & $(9.84,15.91)$ \\
\hline Urban & 856 & 6688.6 & 19.43 & $(15.37,23.48)$ & 708 & 6157.1 & 7.37 & $(4.73,10.02)$ & 13.65 & (II.0I, 16.3) \\
\hline \multicolumn{11}{|l|}{ Region } \\
\hline Northern & 200 & I 412.9 & 18.67 & $(\mathrm{I} I .62,25.73)$ & 172 & | 265.8 & 7.8 & $(3.41,12.19)$ & 13.54 & $(9.45,17.62)$ \\
\hline Center & 628 & 3462.7 & 15.95 & $(\mathrm{II} .78,20 . \mathrm{II})$ & 387 & 2402.8 & 9.86 & $(5.48,14.23)$ & 13.45 & $(10.7,16.21)$ \\
\hline Mexico City & 44 & I 419.3 & 13.5 & $(3.3,23.70)$ & 37 & I 123.0 & no obs & - & 7.53 & $(1.47,13.6)$ \\
\hline Southern & 463 & 3594.1 & 21.93 & $(16.5,27.37)$ & 436 & 3724.5 & 9.07 & $(5.32,12.82)$ & 15.39 & $(\mathrm{II} .57,19.2)$ \\
\hline \multicolumn{11}{|c|}{ Socioeconomic status (tertile) } \\
\hline I (Low) & 585 & 4270.1 & 19.43 & $(14.84,24.03)$ & 408 & 3278.9 & 9.6 & $(5.76,13.4)$ & 15.15 & $(12.09,18.21)$ \\
\hline 2 (Medium) & 464 & 3296.3 & 16.3 & $(I I .22,21.37)$ & 366 & 2843.5 & 7.81 & $(4.24,11.4)$ & 12.37 & $(9.03,15.70)$ \\
\hline 3 (High) & 283 & 2288.2 & 18.14 & $(\mathrm{II} .8 \mathrm{I}, 24.46)$ & 256 & 2385.4 & 5.4 & $(2.30,8.5 \mathrm{I})$ & 11.64 & $(8.14,15.14)$ \\
\hline \multicolumn{11}{|l|}{ Ethnicity } \\
\hline Indigenous & 97 & 471.6 & 20.61 & $(09.34,31.88)$ & 68 & 460.4 & 6.2 & $(-1.32,|3.7|)$ & 13.5 & $(6.82,20.16)$ \\
\hline Non indigenous & 1256 & 9432.2 & 18.12 & $(14.93,21.32)$ & 977 & 8078.4 & 8 & $(5.70,10.27)$ & 13.44 & $(11.29,15.6)$ \\
\hline \multicolumn{11}{|c|}{ Oportunidades affiliation } \\
\hline Yes & 488 & 2969.7 & 13.5 & $(9.46,17.5)$ & 312 & 2308.0 & 12.04 & $(7.18,16.90)$ & 12.85 & $(9.75,15.94)$ \\
\hline No & 845 & 6912.2 & 20.12 & $(16.03,24.21)$ & 717 & 6181.9 & 6.1 & $(3.76,8.44)$ & 13.5 & $(10.92,16.08)$ \\
\hline \multicolumn{11}{|c|}{ Tissue iron deficiency as by the concentration of soluble transferrin receptors $>6 \mathrm{mg} / \mathrm{Ln}=2398$} \\
\hline Overall national & I 354 & 9894.1 & 7.42 & $(5.37,9.48)$ & 1044 & 8533.8 & 6.42 & $(4.1 I, 8.72)$ & 6.96 & $(5.6 \mathrm{I}, 8.6 \mathrm{I})$ \\
\hline \multicolumn{11}{|l|}{ Group age } \\
\hline $12-14$ & 618 & 4235.2 & 3.43 & $(1.80,5.06)$ & 501 & 4057.6 & 12.46 & $(7.89,17.03)$ & 7.85 & $(5.40,10.30)$ \\
\hline $15-19$ & 736 & 5659.0 & $|0.4|$ & $(7.04,13.78)$ & 543 & 4476.2 & 0.93 & $(0.26,1.6 \mathrm{I})$ & 6.23 & $(4.30,8.15)$ \\
\hline \multicolumn{11}{|l|}{ Dwelling } \\
\hline Rural & 477 & 3169.0 & 6.47 & $(3.47,9.47)$ & 323 & 2347.4 & 5.6 & $(1.79,9.42)$ & 6.1 & $(3.80,8.40)$ \\
\hline Urban & 859 & 6710.1 & 7.86 & $(5.19,10.53)$ & 708 & 6163.8 & 6.7 & $(3.92,9.58)$ & 7.33 & $(5.43,9.22)$ \\
\hline \multicolumn{11}{|l|}{ Region } \\
\hline Northern & 199 & 1411.6 & 7.77 & $(3.59,11.95)$ & 173 & | 275. I & 4.67 & $(1.23,8.11)$ & 6.3 & $(3.52,9.07)$ \\
\hline Center & 628 & 3462.7 & 7.98 & $(4.30,11.67)$ & 387 & 2402.8 & 7.77 & $(3.59,11.95)$ & 6.68 & $(4.26,9.09)$ \\
\hline Mexico City & 44 & I 419.3 & 7.55 & $(0.2,14.9)$ & 37 & I 123.0 & 14 & $(2.92,25.01)$ & 10.4 & $(4.05,16.72)$ \\
\hline Southern & 465 & 3585.6 & 6.66 & $(3.78,9.55)$ & 434 & 3710.2 & 5.82 & $(2.82,8.82)$ & 6.24 & $(4.15,8.32)$ \\
\hline \multicolumn{11}{|c|}{ Socioeconomic status (tertile) } \\
\hline I (Low) & 584 & 4242.7 & 9.74 & $(6.08,13.4)$ & 405 & 3260.0 & 5.54 & $(2.17,8.91)$ & 7.92 & $(5.39,10.44)$ \\
\hline 2 (Medium) & 465 & 3303.3 & 5.2 & $(2.15,8.25)$ & 367 & 2852.8 & 8.3 & $(3.84,12.73)$ & 6.63 & $(4.03,9.22)$ \\
\hline 3 (High) & 284 & 2298.8 & 6.41 & $(2.55,10.27)$ & 257 & 2390.0 & 5.45 & $(0.80,10.1 \mathrm{I})$ & 5.92 & $(2.82,9.02)$ \\
\hline \multicolumn{11}{|l|}{ Ethnicity } \\
\hline Indigenous & 94 & 436.7 & 6.86 & $(1.57,12.15)$ & 67 & 448.8 & 4.58 & $(-1.57,10.73)$ & 5.7 & $(I .69,9.7 I)$ \\
\hline Non indigenous & 1260 & 9457.4 & 7.45 & $(5.32,9.58)$ & 977 & 8085.0 & 6.52 & $(4.11,8.92)$ & 7.02 & $(5.46,8.58)$ \\
\hline \multicolumn{11}{|c|}{ Oportunidades affiliation } \\
\hline Yes & 487 & 2949.4 & 4 & $(2.08,5.92)$ & 311 & 2296.4 & 7.81 & $(3.19,12.43)$ & 5.66 & $(3.33,8.01)$ \\
\hline No & 847 & 6922.9 & 8.88 & $(6.07, \mathrm{II} .68)$ & 717 & 6188.5 & 6 & $(3.26,8.63)$ & 7.5 & $(5.6,9.4)$ \\
\hline
\end{tabular}

* $\mathrm{N}$ (thousands) is the expansion for overall population expressed in thousands 
Table III

Prevalence of low serum copper, magnesium and zinc in Adolescents.

\section{ENSANUT 2006}

\begin{tabular}{cc} 
Females \\
\hline $\begin{array}{c}\text { Expansion* } \\
n \\
n\end{array}$ Prevalence $\quad(95 \% \mathrm{Cl})$ \\
Sample (Thousands)
\end{tabular}

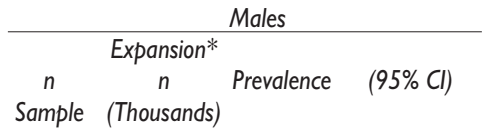

Overall $\quad(95 \% \mathrm{Cl})$

\begin{tabular}{|c|c|c|c|c|c|c|c|c|c|c|}
\hline \multicolumn{9}{|c|}{ Low serum copper $<70$ ug $/ \mathrm{dL}$ males; $<80$ ug/dL females } & \multicolumn{2}{|c|}{$n=1921$} \\
\hline Overall national & 922 & $8,289.4$ & 15.24 & $(12.05,18.43)$ & 999 & $8,441.7$ & 13.05 & $(9.77,16.32)$ & 14.14 & $(11.96,16.63)$ \\
\hline \multicolumn{11}{|l|}{ Dwelling } \\
\hline Rural & 268 & $2,167.2$ & 14.42 & $(9.09,19.76)$ & 301 & $2,300.0$ & 12.25 & $(6.15,18.34)$ & 13.3 & $(9.11,17.5)$ \\
\hline Urban & 713 & $6,111.6$ & 15.56 & $(I I .67,19.44)$ & 685 & $6,119.0$ & 13.4 & $(9.50,17.3)$ & 14.47 & $(I 1.68,17.27)$ \\
\hline \multicolumn{11}{|l|}{ Region } \\
\hline Northern & 201 & I,427.4 & 25.37 & $(18.49,32.25)$ & 175 & $1,290.9$ & 29.1 & $(18.95,39.24)$ & 27.14 & $(20.65,33.63)$ \\
\hline Center & 319 & I,938.5 & 14.01 & $(8.27,19.74)$ & 364 & $2,328.8$ & 10.01 & $(4.78,15.24)$ & 11.83 & $(7.90,15.75)$ \\
\hline Mexico City & 42 & I,354.2 & 13.92 & $(3.14,24.70)$ & 36 & $\mathrm{I}, 107.5$ & 15.1 & $(1.89,28.3)$ & 14.45 & $(6.53,22.37)$ \\
\hline Southern & 419 & $3,558.7$ & 12.4 & $(8.08,16.72)$ & $4 I I$ & $3,691.8$ & 8.82 & $(5.08,12.55)$ & 10.6 & $(7.7|| 3.44)$, \\
\hline
\end{tabular}

Socioeconomic

Status (tertile)

\begin{tabular}{lllllllllll}
$\mathrm{I}($ Low $)$ & 396 & $3,302.9$ & 15.03 & $(\mathrm{I} 0.18,19.87)$ & 390 & $3,248.7$ & 8.4 & $(4.60,12.2 \mathrm{I})$ & $\mathrm{II} .74$ & $(8.66,14.83)$ \\
\hline 2 (Medium) & 338 & $2,840.8$ & 17.68 & $(\mathrm{II} .50,23.86)$ & 350 & $2,805.7$ & 14.35 & $(8.89,19.8 \mathrm{I})$ & 16.02 & $(\mathrm{II} .82,20.23)$ \\
\hline 3 (High) & 244 & $2,100.7$ & 12.6 & $(7.79,17.40)$ & 244 & $2,356.2$ & 17.96 & $(\mathrm{II} .17,24.75)$ & 15.43 & $(\mathrm{I} 0.79,20.07)$
\end{tabular}

Ethnicity

\begin{tabular}{lrrrrrrrrrr} 
indigenous & 65 & 369.3 & 8.6 & $(-1.15,18.35)$ & 68 & 473.2 & 5.71 & $(-0.45,11.88)$ & 7 & $(1.59,12.36)$ \\
\hline Non indigenous & 927 & $7,920.1$ & 15.55 & $(12.25,18.85)$ & 931 & $7,968.5$ & 13.48 & $(10.04,16.9)$ & 14.51 & $(12.08,16.94)$ \\
& & & & & & & & & & \\
$\begin{array}{l}\text { Oportunidades affiliation } \\
\text { Yes }\end{array}$ & 260 & $1,952.3$ & 17.09 & $(10.69,23.48)$ & 294 & $2,282.6$ & 10.63 & $(5.89,15.36)$ & 13.61 & $(9.35,17.86)$ \\
\hline No & 718 & $6,316.6$ & 14.64 & $(11.11,18.17)$ & 690 & $6,118.1$ & 14.04 & $(10.24,17.84)$ & 14.34 & $(11.73,16.95)$
\end{tabular}

Zinc deficiency (<65 ug/dL)

\begin{tabular}{lllllllllll}
$\begin{array}{l}\text { Overall national } \\
\text { Dwelling } \\
\text { Rural }\end{array}$ & 1,019 & $7,670.8$ & 28.09 & $(23.72,32.92)$ & 734 & $6,481.7$ & 24.5 & $(19.86,29.83)$ & 26.45 & $(22.82,30.42)$ \\
\hline Urban & 527 & $49,815.8$ & 25.74 & $(20.35,31.98)$ & 646 & $5,299.6$ & 29.97 & $(24.42,36.18)$ & 27.92 & $(23.58,32.72)$ \\
\hline & 206 & $14,774.2$ & 20.72 & $(12.68,31.99)$ & 371 & $2,366.9$ & 23.83 & $(17.68,31.31)$ & 22.64 & $(16.59,30.09)$
\end{tabular}

Region

\begin{tabular}{lrrrrrrrrrr} 
Northern & 169 & $12,547.2$ & 27.59 & $(19.64,37.27)$ & 189 & $1,346.9$ & 29.43 & $(21.16,39.31)$ & 28.54 & $(21.84,36.34)$ \\
\hline Center & 340 & $21,487.5$ & 22.05 & $(15.36,30.61)$ & 584 & $3,151.6$ & 28.22 & $(22.18,35.17)$ & 25.72 & $(20.42,31.84)$ \\
\hline Mexico City & 35 & $10,633.7$ & 27.63 & $(13.53,48.23)$ & 42 & $1,344.8$ & 31.20 & $(17.85,48.61)$ & 29.62 & $(18.77,43.4)$ \\
\hline Southern & 189 & $19,921.7$ & 23.81 & $(16.29,33.42)$ & 202 & $1,823.1$ & 24.52 & $(17.09,33.86)$ & 24.15 & $(17.76,31.94)$
\end{tabular}

\begin{tabular}{|c|c|c|c|c|c|c|c|c|c|c|}
\hline \multicolumn{11}{|l|}{ Socioeconomic } \\
\hline I (Low) & 255 & $20,751.3$ & 19.97 & $(13.82,27.96)$ & 424 & $3,020.7$ & 27.62 & $(21.11,35.24)$ & 24.50 & $(19.04,30.93)$ \\
\hline 2 (Medium) & $27 \mid$ & $22,833.8$ & 20.78 & $(14.72,28.49)$ & 369 & $2,7 \mid 5.4$ & 26.73 & $(20.08,34.62)$ & 24.01 & $(19.2,29.58)$ \\
\hline 3 (High) & 206 & $20,948.2$ & 33.39 & $(24.96,43.03)$ & 222 & I,898.9 & 31.19 & $(23.14,40.58)$ & 32.34 & $(26.19,39.18)$ \\
\hline
\end{tabular}




\begin{tabular}{|c|c|c|c|c|c|c|c|c|c|c|}
\hline \multicolumn{11}{|l|}{ (Continued) } \\
\hline \multicolumn{11}{|l|}{ Ethnicity } \\
\hline Indigenous & 29 & $2,273.4$ & 18.39 & $(7.01,40.23)$ & 44 & 169.5 & 28.23 & $(10.43,57.08)$ & 22.59 & $(10.72,41.5)$ \\
\hline Non indigenous & 705 & $62,543.3$ & 24.73 & $(19.95,30.21)$ & 975 & $7,501.3$ & 28.08 & $(23.65,32.98)$ & 26.56 & $(22.85,30.63)$ \\
\hline \multicolumn{11}{|c|}{ Oportunidades affiliation } \\
\hline Yes & 192 & $14,536.4$ & 25.43 & $(16.49,37.06)$ & 355 & $2,184.9$ & 27.26 & $(19.74,36.35)$ & 26.53 & $(19.67,34.75)$ \\
\hline No & 539 & $49,870.2$ & 24.10 & $(18.99,30.08)$ & 660 & $5,473.6$ & 28.44 & $(23.39,34.09)$ & 26.37 & $(22.49,30.66)$ \\
\hline \multicolumn{9}{|c|}{ Low serum magnesium $<1.82 \mathrm{mg} / \mathrm{dL}$} & \multicolumn{2}{|c|}{$n=1972$} \\
\hline Overall national & 982 & $8,289.4$ & 40.02 & $(35.21,45.03)$ & 990 & $8,462.4$ & 35.39 & $(30.68,40.4 I)$ & 37.68 & $(33.64,41.91)$ \\
\hline \multicolumn{11}{|l|}{ Dwelling } \\
\hline Rural & 268 & $2,167.2$ & 41.93 & $(32.74,51.71)$ & 302 & $2,302.8$ & 32.50 & $(25.05,40.96)$ & 37.07 & $(29.64,45.17)$ \\
\hline Urban & 713 & $6,111.6$ & 39.24 & $(33.64,45.13)$ & 687 & $6,137.0$ & 36.61 & $(30.82,42.82)$ & 37.92 & $(33.16,42.93)$ \\
\hline \multicolumn{11}{|l|}{ Region } \\
\hline Northern & 201 & $\mathrm{I}, 427.4$ & 52.55 & $(43.26,61.66)$ & 175 & $1,290.9$ & 44.70 & $(34.4,55.47)$ & 48.82 & $(40.54,57.17)$ \\
\hline Center & 319 & $1,938.5$ & 35.58 & $(27.86,44.15)$ & 366 & $2,334.1$ & 33.64 & $(26.24,41.95)$ & 34.52 & $(27.78,41.96)$ \\
\hline Mexico City & 42 & $1,354.2$ & 41.48 & $(26.8,57.85)$ & 37 & $1,123.0$ & 43.19 & $(26.87,61.13)$ & 42.25 & $(30.98,54.4)$ \\
\hline Southern & 419 & $3,558.7$ & 36.67 & $(29.49,44.5)$ & $4 I I$ & $3,691.8$ & 31.09 & $(24.18,38.97)$ & 33.83 & $(27.5,40.8)$ \\
\hline \multicolumn{11}{|c|}{ Socioeconomic status (tertile) } \\
\hline I(Low) & 396 & $3,302.9$ & 41.27 & $(34.34,48.56)$ & 391 & $3,251.5$ & 31.23 & $(24.49,38.86)$ & 36.29 & $(30.6,42.38)$ \\
\hline 2 (Medium) & 338 & $2,840.8$ & 45.30 & $(37.5,53.33)$ & 351 & $2,821.1$ & 40.56 & $(33.4,48.15)$ & 42.94 & $(37.01,49.07)$ \\
\hline 3 (High) & 244 & $2,100.7$ & 31.13 & $(23.48,39.97)$ & 245 & $2,358.7$ & 35.31 & $(26.93,44.7)$ & 33.34 & $(27.03,40.3)$ \\
\hline \multicolumn{11}{|l|}{ Ethnicity } \\
\hline Indigenous & 55 & 369.3 & 47.55 & $(26.11,69.93)$ & 56 & 473.2 & 25.93 & $(11.85,47.69)$ & 35.41 & $(19.54,55.31)$ \\
\hline Non indigenous & 927 & $7,920.1$ & 39.67 & $(34.75,44.8 I)$ & 934 & $7,989.2$ & 35.95 & $(3 I . I, 4 I . I I)$ & 37.80 & $(33.66,42.13)$ \\
\hline \multicolumn{11}{|c|}{ Oportunidades affiliation } \\
\hline Yes & 260 & $1,952.3$ & 39.54 & $(30.85,48.94)$ & 294 & $2,282.6$ & 30.37 & $(23.2,38.64)$ & 34.60 & $(27.81,42.08)$ \\
\hline No & 718 & $6,316.6$ & 39.97 & $(34.44,45.77)$ & 693 & $6,138.8$ & 37.50 & $(31.84,43.52)$ & 38.75 & $(34.1,43.62)$ \\
\hline
\end{tabular}

$* N$ (thousands) is the expansion for overall population expressed in thousands

prevalence was significantly higher in the Northern region $(27.14 \%)$ than in the Center (11.8\%) or the Southern $(10.6 \%)$ regions; it was also lower in indigenous (7\%) than in non indigenous (14.5\%) subjects (Table III).

The median daily intake of copper was $0.90 \mathrm{mg} / \mathrm{d}$, with an overall adequacy of $140 \% ; 162 \%$ for males and $127.7 \%$ for females.

In a logistic regression model, the risk to suffer low serum copper increased with age in males $(\mathrm{OR}=1.18$, $p=0.01)$, but not in females $(\mathrm{OR}=0.96, p=0.58)$. Living in the Northern region $(\mathrm{OR}=2.79, p<0.001)$ represented a risk for low serum copper. No significant differences were observed by dwelling, SES or affiliation to Oportunidades (Table IV). Intake of copper, phytic acid and fiber (as tertiles) were not associated with LSC (data not shown).

\section{Zinc deficiency}

The overall mean of serum concentration of zinc was $92.82 \pm 1.02 \mu \mathrm{g} / \mathrm{dL}, 95.43 \pm 1.03 \mu \mathrm{g} / \mathrm{dL}$ for males and $90.72 \pm 1.03 \mu \mathrm{g} / \mathrm{dL}$ for females. The overall prevalence of zinc deficiency was $26.4 \%$ (Table III).

The median of dietary intake of zinc was significantly higher for males $(8.1 \mathrm{mg} / \mathrm{d})$ than females $(6.4$ $\mathrm{mg} / \mathrm{d}$ ), with an adequacy of $93 \%$.

In a logistic regression model, no significant associations were found among zinc deficiency and sex, 
Table IV

LOGISTIC REGRESSION MODELS FOR PREDICTED VARIABLES OF MICRONUTRIENTS DEFICIENCY. ENSANUT 2006

\begin{tabular}{|c|c|c|c|c|c|c|c|c|c|c|}
\hline \multirow{2}{*}{$\begin{array}{l}\text { Depent variable } \\
\text { Sex }\end{array}$} & \multicolumn{2}{|c|}{$\begin{array}{c}\text { Model I } \\
\text { Low iron stores } \\
(\text { Ferritin }<12 \mu \mathrm{g} / \mathrm{L})\end{array}$} & \multicolumn{2}{|c|}{$\begin{array}{l}\text { Model } 2 \\
\text { Tissue iron deficiency } \\
(T f R>6 \mathrm{mg} / \mathrm{L})\end{array}$} & \multicolumn{2}{|c|}{$\begin{array}{c}\text { Model } 3 \\
\text { Low serum copper } \\
(<70 \mu \mathrm{g} / \mathrm{dL} \text { males; }<80 \mu \mathrm{g} / \mathrm{dL} \text { females })\end{array}$} & \multicolumn{2}{|c|}{$\begin{array}{l}\text { Model } 4 \\
\text { Zinc deficiency } \\
(<65 \mu \mathrm{g} / \mathrm{dL})\end{array}$} & \multicolumn{2}{|c|}{$\begin{array}{l}\text { Model } 5 \\
\text { Low serum magnesium } \\
(<1.82 \mathrm{mg} / \mathrm{dL})\end{array}$} \\
\hline & OR & $P$ value & OR & $P$ value & OR & $P$ value & OR & $P$ value & OR & $P$ value \\
\hline Male & 1.00 & - & 1.00 & - & 1.00 & - & 1.00 & - & 1.00 & - \\
\hline Female & 0.03 & 0.01 & 2.86 e- 6 & $<0.001$ & 22.54 & 0.01 & 1.29 & 0.13 & 1.14 & 0.28 \\
\hline Age (years) & 0.82 & 0.02 & 0.49 & $<0.001$ & 1.18 & 0.01 & 0.97 & 0.33 & 1.01 & 0.83 \\
\hline Sex $*$ age interaction & 1.36 & $<0.001$ & 2.43 & $<0.001$ & 0.82 & 0.02 & - & - & - & - \\
\hline \multicolumn{11}{|l|}{ Dwelling } \\
\hline Urban & 1.00 & - & 1.00 & - & 1.00 & - & 1.00 & - & 1.00 & - \\
\hline Rural & 0.72 & 0.10 & 0.89 & 0.69 & 1.06 & 0.85 & 0.72 & 0.25 & 1.18 & 0.43 \\
\hline
\end{tabular}

Region

\begin{tabular}{|c|c|c|c|c|c|c|c|c|c|c|}
\hline Centre & 1.00 & - & 1.00 & - & 1.00 & - & 1.00 & - & 1.00 & - \\
\hline Northern & 1.05 & 0.85 & 0.97 & 0.94 & 2.79 & 0.00 & 1.16 & 0.55 & 1.82 & 0.02 \\
\hline Mexico City & 0.42 & 0.08 & 1.48 & 0.39 & 1.30 & 0.5 & 1.22 & 0.57 & 1.41 & 0.28 \\
\hline Southern & 1.22 & 0.36 & 0.82 & 0.48 & 0.86 & 0.57 & 0.93 & 0.79 & 1.07 & 0.79 \\
\hline Body Mass Index (kg/m2) & 0.97 & 0.17 & 1.06 & 0.02 & 0.96 & 0.07 & - & - & 1.03 & 0.06 \\
\hline C-Reactive protein $(\mathrm{mg} / \mathrm{dL})$ & 0.92 & 0.03 & - & - & 0.86 & 0.08 & 1.01 & 0.42 & 1.00 & 0.64 \\
\hline
\end{tabular}

Ethnicity

\begin{tabular}{lcccccccccccc} 
Non indigenous & 1.00 & - & 1.00 & - & 1.00 & - & 1.00 & - & 1.00 & - \\
\hline Indigenous & 0.82 & 0.58 & 0.74 & 0.54 & 0.61 & 0.28 & 1.09 & 0.86 & 1.13 & 0.81
\end{tabular}

Oportunidades affiliation

\begin{tabular}{ccccccccccccccc} 
No & 1.00 & - & 1.00 & - & 1.00 & - & 1.00 & - & 1.00 & - & 0 \\
\hline Yes & 0.91 & 0.65 & 0.69 & 0.24 & 1.50 & 0.15 & 1.36 & 0.20 & 0.82 & 0.33 &
\end{tabular}

Socioeconomic status

\begin{tabular}{lccccccccccc} 
Tertile I (Low) & 1.00 & - & 1.00 & - & 1.00 & - & - & 1.00 & - & 1.00 & - \\
\hline Tertile 2 (Middle) & 0.73 & 0.11 & 0.61 & 0.10 & 1.23 & 0.35 & 0.92 & 0.71 & 1.18 & 0.34 \\
\hline Tertile 3 (High) & 0.76 & 0.24 & 0.49 & 0.07 & 1.20 & 0.48 & 1.44 & 0.13 & 0.73 & 0.13
\end{tabular}

dwelling, region, age, height-for- age z-score, CRP, intake of fiber or phytic acid, zinc, ethnicity, SES or affiliation to Oportunidades. (Table IV). Zinc deficiency was no associated with dietary intake of zinc, phytic acid and fiber (data not shown).

\section{Low serum magnesium}

The mean serum concentration of magnesium was $1.92 \pm 1.01 \mathrm{mg} / \mathrm{dL}$, with no differences by sex. The overall prevalence of low serum magnesium was $37.6 \%$, being higher in the Northern $(48.8 \%)$ than the Southern region $(33.8 \%)$ (Table III).
The overall median daily intake of magnesium was $234.9 \mathrm{mg} / \mathrm{d}$, slightly higher for male than for female (268.5 mg/d vs. $215.7 \mathrm{mg} / \mathrm{d}$, respectively), with a median adequacy of $85 \%$.

In a logistic regression model, living in the Northern region was a risk for low serum magnesium (LSM) $(\mathrm{OR}=1.82, p=0.02)$. No significant associations were found with sex, dwelling, BMI, CRP, SES, ethnicity or affiliation to Oportunidades (Table IV). LSM was no associated with dietary intake of magnesium, phytic acid and fiber (data not shown). 


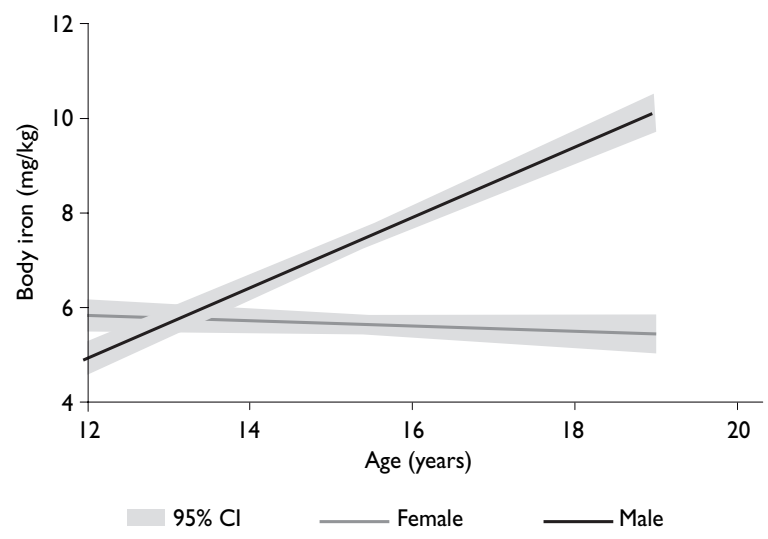

Figure I. EFFECT OF AGE ON TOTAL BOdY IRON BY SEX IN Mexican ADOLESCENTS. ENSANUT 2006

\section{Discussion}

We present evidence herein for a high prevalence of iron, zinc, copper and magnesium deficiencies in Mexican male and female adolescents. Females were more prone to suffer most of mineral deficiencies. When comparing the prevalence of LIS in female adolescents in the NNS-99 with those herein reported, there is a decrement in the prevalence (40.5\% vs $18.4 \%) .{ }^{9}{ }^{20}$ However, methodological differences may confound such a big decrease; in 1999 LIS was assessed by percent saturation of transferrin, while in 2006 a direct measurement of s-ferritin was used.

The puberty onset is probably critical to enhance the sexual differences in TBI in this study, i.e. a larger growth of muscle mass in males ${ }^{21}$ and losses of iron throughout menstruation results in larger TBI in males relative to females. Information on population based estimates of TBI are scanty; data from NHANES III reported in adults older than 20 years mean TBI values of $9.89 \pm 2.82 \mathrm{mg} / \mathrm{kg}$ for males and $4.87 \pm 4.14 \mathrm{mg} / \mathrm{kg}$ for females, ${ }^{14}$ similar to those reported herein. We are aware that Cook's equation is not validated for adolescents, however, even if the external validity may be questionable, the internal validity allows for valid comparisons between both genders. We made efforts to homologate our TfR results to Cook's laboratory, correcting as by Ramco's kit.

In the present study, the majority of cases of anemia were not explained by ID; other nutritional causes of anemia as such folate and vitamin B12 deficiencies were not evaluated. ${ }^{22}$ However, the adequacy of the dietary intake of vitamin B12 was $110 \%$, while the adequacy for folate was $70 \%$. This may explain, in part, the high proportion of non IDA. Both, low and excessive copper serum concentrations are associated with IDA., ${ }^{4,23}$ In our study, adolescents with anemia had significantly higher serum concentrations of copper than their non anemia counterparts, in line with the reported by NHANES-II. ${ }^{4}$ The association between iron and copper deficiencies is most probably due to the role of copper as a cofactor of iron oxidizing proteins as such hefastin and ceruloplasmine. ${ }^{24}$

The possibility of subestimating ID as a cause of anemia could be influenced by acute infections and inflammation. However, the rate of acute infections at this age is low. ${ }^{25}$ We used a $6 \mathrm{mg} / \mathrm{L}$ cut-off for CRP, instead of the usual $3 \mathrm{mg} / \mathrm{dL}$, because in a segmented regression model using data from two probabilistic surveys in Mexican population $(n=5000)$ the inflexion point for ferritin values occurred at a concentration of $6 \mathrm{mg} / \mathrm{L}$ of CRP (data not published).

\section{Low serum copper}

The lack of population-based studies on copper status in adolescents prevents from an adequate comparison of our data. The prevalence of low copper concentrations among adolescent girls from Sudan was 5.9\%; ${ }^{26}$ in Iranian pre and postmenopausal women was $17 \%$; 27 in Chilean adults the prevalence was $8.6 \%$ in males and $5.9 \%$ in females. ${ }^{28}$ The prevalence of low serum copper in our series is one of the highest in the literature. The rarity and letality of genetic diseases associated with copper deficiencies as such Menkes disease and aceruloplasminemia ${ }^{29,30}$ make difficult to invoke them as significant causes of this large proportion of LSC. It is possible that the higher copper requirement during the puberty growth spurt drains copper out of the circulation. ${ }^{30}$

\section{Zinc deficiency}

The prevalence of zinc deficiency in female adolescents herein reported was similar to that in NNS-99 (29.7\%). The overall prevalence was higher than reported for developed countries but lower than for developing countries. In American children 9-19 years of age, the prevalence was $1 \%$ for males and $1.3 \%$ for females. ${ }^{31}$ In British children 11-14 and 15-18 years of age the prevalence of zinc deficiency for boys was 0 and $2 \%$, and for girls 2 and 5\% respectively. ${ }^{7}$ Prevalence in Nepalese females $13-35$ years of age, varied from 78 to $90 \% .^{32}$ The Mexican prevalence herein reported is lower than for Southeast Asia (71.2\%), Africa (68\%), East Mediterranean $(73.5 \%)$ and the Americas $(45.8 \%)$ but higher than for the West Pacific (18.6\%) and Europe: $8.0 \%{ }^{1}$ 
The high prevalence of zinc deficiency is relevant for the future health of adolescents because it is associated with higher risks for low birth weight ${ }^{33}$ and impinges on physical growth in small children. ${ }^{34,35}$ It also affects negatively on the cognitive function and emotional performance. ${ }^{36}$

\section{Low serum magnesium}

The prevalence of low serum magnesium in this survey is higher than the reported for German individuals $(14.5 \%),{ }^{3}$ though, they used a higher cut-off than in this study (1.85 mg/dL vs $1.82 \mathrm{mg} / \mathrm{dL}$, respectively), it is not enough to explain such a large difference. It is more probable that an insufficient diet in Mexico, especially in animal tissue may explain such a difference. We were not able to find serum magnesium data from suboptimally nourished countries. The lower prevalence in the Southern region could be due to the observed higher daily intake of magnesium than in the Northern region (data not shown). We ascertained that the high prevalence was not due to an inaccurate magnesium determination, results were calibrated against NIST SRM 3131a reference materials.

Low serum magnesium causes potassium, calcium and neuromuscular disturbances, central nervous system and cardiovascular alterations, like arrhythmias. ${ }^{37}$ Also, can alter glucose homeostasis, increase atherosclerosis, hypertension, myocardial infarction, osteoporosis, migraine, asthma, chronic fatigue syndrome, among others. ${ }^{37-39}$ More research on the effects of magnesium deficiency on the health of adolescents is needed to warrant interventions to prevent it.

The high prevalence of iron deficiency and low serum zinc, magnesium and copper detected in Mexican adolescents could be partially explained by their relatively high intake of antagonists of the absorption of those minerals, as such fiber, tannins and phytates, ${ }^{40}$ and low intake of enhancers of mineral absorption as such vitamin C. ${ }^{41}$ The best sources for iron, zinc and copper are animal tissues scarce in the Mexican diet, and could explain the coexistence of deficiencies of those minerals.

The deficiencies detected by the serum concentrations of zinc, cooper and magnesium can be graded from moderate to severe; slight deficiencies are not detected by this method thus their prevalence might be underestimated.

In summary we document here the prevalence and distribution of several mineral deficiencies including iron, copper, zinc and magnesium in Mexican adolescents. Despite several public nutritional interventions in Mexico provide iron and zinc supplementation, the prevalence of iron and zinc deficiencies are still very high, calling for a more careful examination of the effectiveness of those interventions in order to redesign them aiming for better outcomes.

Declaration of conflict of interest: The authors declare that they have no conflict of interests.

\section{References}

I. Ramakrishnan U. Prevalence of micronutrient malnutrition worldwide. Nutr Rev 2002;60:S46-S52.

2. Viteri FE, Gonzalez H. Adverse outcomes of poor micronutrient status in childhood and adolescence. Nutr Rev 2002;60(5 Pt 2):S77-83.

3. Schimatschek HF, Rempis R. Prevalence of hypomagnesemia in an unselected German population of 16,000 individuals. Magnes Res 200I;I4(4):283-290.

4. Knovich MA, Il'yasova D, Ivanova A, Molnar I. The association between serum copper and anaemia in the adult Second National Health and Nutrition Examination Survey (NHANES II) population. Br J Nutr 2008;99(6): I226-I 229.

5. Hettiarachchi M, Liyanage C, Wickremasinghe R, Hilmers DC, Abrahams SA. Prevalence and severity of micronutrient deficiency: a cross-sectional study among adolescents in Sri Lanka. Asia Pac J Clin Nutr 2006; I5(I):56-63.

6. Kassu A, Yabutani T, Mulu A, Tessema B, Ota F. Serum zinc, copper, selenium, calcium, and magnesium levels in pregnant and nonpregnant women in Gondar, Northwest Ethiopia. Biol Trace Elem Res 2008; I22(2):97-106.

7. Thane CW, Bates C], Prentice A. Zinc and vitamin A intake and status in a national sample of British young people aged 4-I8 y. Eur J Clin Nutr 2004;58(2):363-375.

8. Shamah-Levy T, Villalpando S, Rivera JA, Mejia-Rodriguez F, CamachoCisneros M, Monterrubio EA. Anemia in Mexican women: a public health problem. Salud Publica Mex 2003;45 Suppl 4:S499-507.

9. Villalpando S, Garcia-Guerra A, Ramirez-Silva Cl, Mejia-Rodriguez F, Matute G, Shamah-Levy T, et al. Iron, zinc and iodide status in Mexican children under 12 years and women I2-49 years of age. A probabilistic national survey. Salud Publica Mex 2003;45 Suppl 4:S520-S529.

10. Olaiz-Fernández G, Rivera-Dommarco J, Shamah-Levy T, Rojas R, Villalpando-Hernández S, Hernández-Avila M, eds. Encuesta Nacional de Salud y Nutrición 2006. Cuernavaca, México: Instituto Nacional de Salud Pública, 2006.

II. Lohman T, Roche A, Martorell R. Anthropometric Standardization Reference Manual. Champaign(IL): Human Kinetics Books, I99I.

12. Habicht JP. Standardization of quantitative epidemiological methods in the field. Bol Oficina Sanit Panam 1974;76(5):375-384.

13. Cole TJ, Bellizzi MC, Flegal KM, Dietz WH. Establishing a standard definition for child overweight and obesity worldwide: international survey. BMJ 2000;320(7244):I240-I243.

I4. Cook JD, Flowers CH, Skikne BS. The quantitative assessment of body iron. Blood 2003; I0I(9):3359-3364.

I5. Hess SY, Peerson JM, King JC, Brown KH. Use of serum zinc concentration as an indicator of population zinc status. Food Nutr Bull 2007;28(3 Suppl):S403-S429.

16. Gibson R. Principles of nutritional assessment. Nueva York (NY) ed. O.U. Press, 2005

17. UNICEF/UNU/WHO. Iron deficiency anemia: assessment, prevention, and control. A guide for programme managers. WHO/NHD (200I) [report no. 01.3].

18. Rodríguez-Ramírez S, Mundo-Rosas V, Jiménez-Aguilar A, ShamahLevy T. Methodology for the analysis of dietary data from the Mexican 
National Health and Nutrition Survey 2006. Salud Publica Mex 2009;5 I suppl 4:S523-S529.

19. Dietary reference intakes for energy, carbohydrate, fiber, fat, fatty acids, cholesterol, protein, and amino acids. Washington DC: National Academies Press, 2002.

20. Villalpando S, Shama-Levy T, Mundo V, Rivera-Dommarco J. Análisis crítico de la evolución de la prevalencia de anemia y la deficiencia de micronutrimentos en la población. Salud Publica Mex 2007;49 ed especial: E270-E272.

2I. Rubinstein JH. Broad thumb-hallux (Rubinstein-Taybi) syndrome 1957-1988. Am J Med Genet Suppl 1990;6:3-16.

22. Chandra J. Megaloblastic anemia: back in focus. Indian J Pediatr 2010;77(7):795-799.

23. Huff JD, Keung YK, Thakuri M, Beaty MW, Hurd DD, Owen J, et al. Copper deficiency causes reversible myelodysplasia. Am J Hematol 2007;82(7):625-630.

24. Frazer DM, Anderson G]. Iron imports. I. Intestinal iron absorption and its regulation. Am J Physiol Gastrointest Liver Physiol 2005;289(4):G63I-G635.

25. Martinez-Garcia MC, Munoz O, Peniche A, Ramirez-Grande ME, Gutierrez G. Epidemiology of acute diarrhoea in a rural medical care system in Mexico (IMSS-COPLAMAR). Arch Invest Med (Mex) 1989;20(I):69-78.

26. Weisstaub S, Bustos M, Olivares M, Castillo D, Araya M. Situación nutricional de hierro, cobre y zinc en escolares de Tacopaya, Bolivia. Rev Bol Ped 2004;43(2):77-80.

27. Mir E, Hossein-nezhad A, Bahrami A, Bekheirnia M, Javadi E, AfsharNaderi A, et al. Adequate Serum Copper Concentration Could Improve Bone Density, Postpone Bone Loss and Protect Osteoporosis in Women. Iranian J Public Health 2007:24-29.

28. Cediel G, Olivares M, Araya M, Letelier M, López de Romana D, Pizarra F. Efecto de la inflamación subclínica sobre el estado nutricional de hierro, cobre y zinc en adultos. Rev Chil Nutr 2009;36:8-14.

29. Houwen RH. Copper: two sides of the same coin. Neth J Med 2008;66(8):325-326.
30. Olivares M, Araya M, Uauy R. Copper homeostasis in infant nutrition: deficit and excess. J Pediatr Gastroenterol Nutr 2000;31 (2): I02-III. 3I. Pilch SM, Senti FR. Analysis of zinc data from the second National Health and Nutrition Examination Survey (NHANES II). J Nutr 1985; | I5(II):I393-1397.

32. Chandyo RK, Strand TA, Mathisen M, Ulak M, Adhikari RK, Bolann BJ, et al. Zinc deficiency is common among healthy women of reproductive age in Bhaktapur, Nepal. J Nutr 2009;139(3):594-597.

33. King JC. Determinants of maternal zinc status during pregnancy. Am J Clin Nutr 2000;7I(5 Suppl): I334S-1343S.

34. Brown KH, Peerson JM, Rivera J, Allen LH. Effect of supplemental zinc on the growth and serum zinc concentrations of prepubertal children: a meta-analysis of randomized controlled trials. Am J Clin Nutr 2002;75(6): 1062-1071.

35. Bhutta ZA, Black RE, Brown KH, Gardner JM, Gore S, Hidayat A, et al. Prevention of diarrhea and pneumonia by zinc supplementation in children in developing countries: pooled analysis of randomized controlled trials. Zinc Investigators' Collaborative Group. J Pediatr 1999; 135(6):689-697.

36. Tupe RP, Chiplonkar SA. Zinc supplementation improved cognitive performance and taste acuity in Indian adolescent girls. J Am Coll Nutr 2009;28(4):388-396

37. Swaminathan R. Magnesium metabolism and its disorders. Clin Biochem Rev 2003;24(2):47-66.

38. King DE. Inflammation and elevation of C-reactive protein: does magnesium play a key role? Magnes Res 2009;22(2):57-59.

39. Barbagallo M, Dominguez LI. Magnesium metabolism in type 2 diabetes mellitus, metabolic syndrome and insulin resistance. Arch Biochem Biophys 2007;458(I):40-47.

40. Hambidge KM, Miller LV, Westcott JE, Sheng X, Krebs NF. Zinc bioavailability and homeostasis. Am J Clin Nutr 91(5): I478S- I483S. 4I. Lozada AL, Flores M, Rodríguez S, Barquera S. Patrones dietarios en adolescentes mexicanas. Una comparación de dos métodos. Encuesta Nacional de Nutrición, 1999. Salud Publica Mex 2007;49(4):263-273. 